\title{
Combination Immunosuppressive Therapy for Giant Cell Myocarditis: A Case Report
}

\author{
Takashi Funaki ${ }^{1}$, Mike Saji ${ }^{1}$, Tatsuya Murai ${ }^{2}$, Ryosuke Higuchi ${ }^{1}$, \\ Mamoru Nanasato ${ }^{1}$ and Mitsuaki Isobe $^{3}$
}

\begin{abstract}
:
A 60-year-old woman with a history of hypothyroidism was referred to our hospital for shortness of breath and a left ventricular ejection fraction (LVEF) of $13 \%$, which required continuous dobutamine injection with intra-aortic balloon pump support. An endomyocardial biopsy obtained from the right ventricle revealed an infiltration of giant cells and eosinophils, indicating giant cell myocarditis. In addition to heart failure treatment, combined immunotherapy with steroids, tacrolimus, and intravenous immunoglobulin was administered. Transthoracic echocardiography demonstrated a dramatic improvement in the LVEF after this therapy, and the patient was discharged home without symptoms on day 72 .
\end{abstract}

Key words: Giant cell myocarditis, Intravenous immunoglobulin therapy, Tacrolimus

(Intern Med Advance Publication)

(DOI: 10.2169/internalmedicine.9112-21)

\section{Introduction}

Giant cell myocarditis is a rare disease that can lead to cardiogenic shock and life-threatening ventricular arrhythmias. This disease is caused by inflammation of the myocardium, which is infiltrated by giant cells, eosinophils, and lymphocytes. In addition to providing these patients with optimal medical treatment for heart failure, mechanically assisted circulation may be required in severe cases.

The prognosis for giant cell myocarditis is reportedly very poor, with many patients eventually requiring heart transplantation (1). In addition to steroid therapy, several immunosuppressive therapeutic regimens, including a combination of cyclosporine and azathioprine, have been reported to be effective treatment options for this disorder $(2,3)$. More recently, tacrolimus/mycophenolate-based regimens have been shown to have fewer adverse effects and greater efficacy than cyclosporine/azathioprine-based regimens (3). Furthermore, some studies have shown that intravenous immunoglobulin (IVIG) therapy reduced in-hospital mortality and significantly improved the LVEF in these patients (4). However, information regarding combination immunosuppressive therapies remains limited.

We herein report a patient who received tacrolimus and IVIG therapy in addition to steroids to suppress the inflammation associated with giant cell myocarditis and to improve her cardiac function, thereby facilitating successful withdrawal from mechanically assisted circulation.

\section{Case Report}

A 60-year-old woman with a history of hypothyroidism had been experiencing severe dyspnea for 10 days. She was referred to our hospital for the management of heart failure despite standard treatment.

On her arrival, a physical examination revealed a heart rate of $107 \mathrm{bpm}$, blood pressure of 115/90 $\mathrm{mmHg}$, and oxygen saturation of $89 \%$ on room air. Laboratory evaluations showed elevated levels of lactic acid at $9.4 \mathrm{mmol} / \mathrm{L}$, creatine kinase (CK) at $802 \mathrm{IU} / \mathrm{L}, \mathrm{CK}-\mathrm{MB}$ isoenzyme at $44.9 \mathrm{IU} / \mathrm{L}$, C-reactive protein at $0.67 \mathrm{mg} / \mathrm{dL}, \mathrm{N}$-terminal pro-brain natriuretic peptide at $53,482 \mathrm{pg} / \mathrm{mL}$, and troponin $\mathrm{T}$ level at $1.020 \mathrm{ng} / \mathrm{mL}$. The thyroid-stimulating hormone and free $\mathrm{T} 4$ levels were normal and did not fluctuate during the treatment progress. She had been diagnosed with hypothyroidism

${ }^{1}$ Department of Cardiology, Sakakibara Heart Institute, Japan, ${ }^{2}$ Department of Pathology, Sakakibara Heart Institute, Japan and ${ }^{3}$ Sakakibara Heart Institute, Japan

Received: November 30, 2021; Accepted: January 23, 2022; Advance Publication by J-STAGE: March 5, 2022

Correspondence to Dr. Mike Saji, mikesaji8@gmail.com 


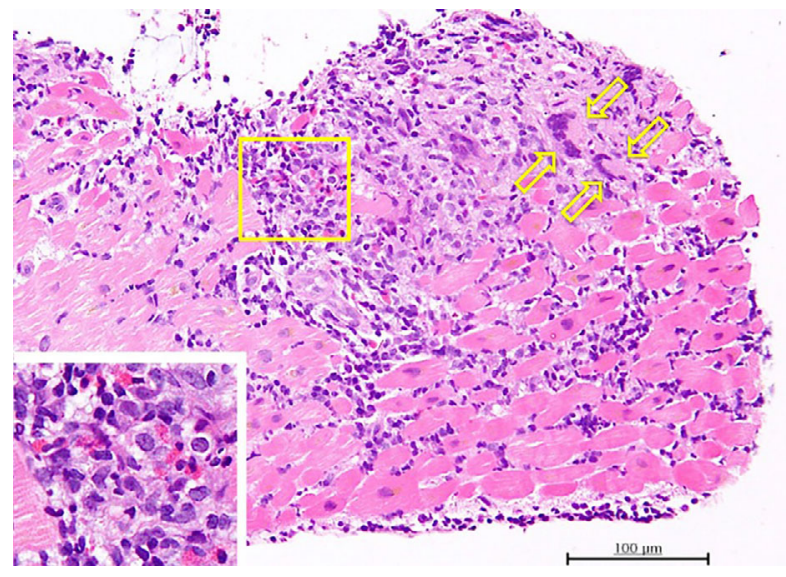

Figure 1. Histological findings from the right ventricular apex, showing an infiltration of multinucleated giant cells (yellow arrow). In the lower left frame (enlarged view of yellow frame), the place where eosinophils are gathered is greatly enlarged. Hematoxylin and Eosin staining.

about 10 years prior to presentation and her antibody levels had thus been high ever since. An echocardiogram revealed severe global systolic dysfunction with a left ventricular (LV) ejection fraction (LVEF) of $13 \%$ and LV thrombus.

Subsequently, we initiated treatment for heart failure with dobutamine and heparin injections; however, she did not adequately respond to these treatments. Consequently, an intra-aortic balloon pump (IABP) was inserted on day 1. At the same time, a coronary angiogram was performed, without evidence of coronary artery stenosis. A Swan Ganz catheter was inserted to evaluate hemodynamics. Right heart catheterization showed a pulmonary capillary wedge pressure of $15 \mathrm{mmHg}$, mean pulmonary arterial pressure of 26 $\mathrm{mmHg}$, and central venous pressure of $13 \mathrm{mmHg}$. We could not perform a myocardial biopsy because the patient presented after regular consulting hours and there were various limitations on both the staff and equipment. As a result of the IABP support, the lactic acid level dropped to $1.0 \mathrm{mmol} /$ $\mathrm{L}$, and the hemodynamics stabilized.

Enalapril (2.5 $\mathrm{mg})$ and spironolactone $(25 \mathrm{mg})$ were started on day 2. Transthoracic echocardiography showed a slight improvement in the LVEF, and the IABP was removed on day 5. However, contrary to our expectations, her hemodynamics once again became unstable on day 6 . Therefore, the IABP was reinserted, and an endomyocardial biopsy was conducted to clarify the cause of the severe heart failure.

The biopsy findings revealed necrosis of myocytes, with inflammatory infiltration of lymphocytes, eosinophils, and giant cells suggestive of giant cell myocarditis (Fig. 1). In addition to the optimal orally administered heart failure treatments, the patient was also started on a 3-day course of intravenous methylprednisolone (1,000 mg daily) followed by a maintenance dose of $50 \mathrm{mg}$ of prednisolone daily; a 2day course of IVIG (50 g/day); and tacrolimus. The tacrolimus was adjusted to a trough value of less than 10 $\mathrm{ng} / \mathrm{mL}$. We started tacrolimus $2 \mathrm{mg}$ daily and increased the dose to $4 \mathrm{mg}$ daily after 5 days of administration because the trough value was $3.3 \mathrm{ng} / \mathrm{dL}$. After treatment with this immunosuppressive regimen, her hemodynamics stabilized again, her mixed venous oxygen saturation $(\mathrm{SvO} 2)$ almost exceeded $60 \%$, and her cardiac index $(\mathrm{CI})$ was $>2.2$. Her troponin T level dropped from $1.020 \mathrm{ng} / \mathrm{mL}$ to $0.147 \mathrm{ng} / \mathrm{mL}$.

The IABP was subsequently removed on day 16 (Fig. 2). Dobutamine was ended on day 49, and carvedilol $10 \mathrm{mg}$, eplerenone $25 \mathrm{mg}$, enalapril $2.5 \mathrm{mg}$, and furosemide $10 \mathrm{mg}$ were administered at discharge. Finally, the patient was discharged home on day 72 without any side effects from steroid or tacrolimus administration and after a subcutaneous implantable cardioverter defibrillator was placed. Transthoracic echocardiography before discharge showed an LVEF of $42 \%$. Another endomyocardial biopsy was conducted to histologically confirm the response of the myocarditis to the combination treatment, with the biopsy findings demonstrating the absence of a giant cell or eosinophil infiltration and only mild residual lymphocyte infiltration (Fig. 3).

\section{Discussion}

Giant cell myocarditis primarily affects healthy individuals; however, noncardiac autoimmune disorders (inflammatory bowel disease, thyroiditis, and thymoma) have been reported in approximately $20 \%$ of cases (2). In the present case, Hashimoto's disease was considered to have been associated with the development of giant cell myocarditis. However, it is unclear under what conditions giant cell myocarditis develops in Hashimoto's disease, so the accumulation of further cases will be necessary. In the present case at least, Hashimoto's disease was stable throughout the course.

Giant cell myocarditis is a rare but very severe myocarditis with a poor prognosis. Previous studies have shown that patients with giant cell myocarditis have a median survival of only 3 months from the onset of symptoms if they do not undergo treatment and of only 3.8 months with steroid treatment alone. However, per a previous report, the median survival time can be extended to 11.5 months with combined cyclosporine and azathioprine treatment (1). Another study analyzed 26 patients treated with combined immunosuppression (2-4 drugs) and showed that the rate of a transplant-free survival from the symptom onset was $69 \%, 58 \%$, and $52 \%$ at 1,2 , and 5 years, respectively (5). Multidrug immunosuppressive therapy is therefore considered an effective treatment option for giant cell myocarditis (1). Our treatment regimen for this disorder includes $1 \mathrm{~g} /$ day of methylprednisolone for 3 days, which is then switched to $1 \mathrm{mg} / \mathrm{kg}$ of prednisolone, with gradual reductions in the dose. In addition to this treatment, the administration of calcineurin inhibitors, such as cyclosporine or tacrolimus, and the combined administration of azathioprine or mycophenolate mofetil may also be considered. The humanized anti-CD52 monoclonal antibody (alemtuzumab) or antithymocyte globulin can alternatively be administered (2). 


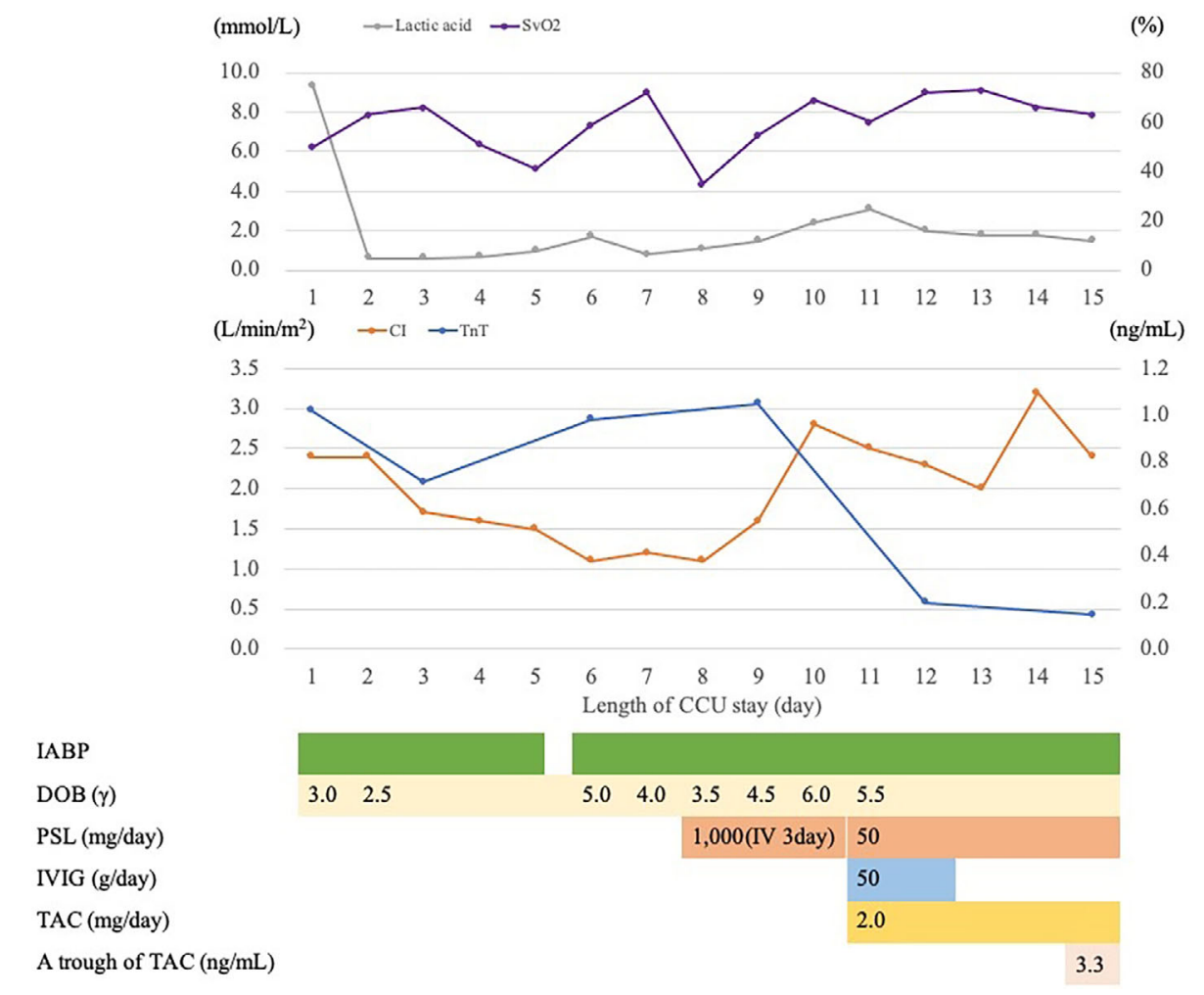

Figure 2. Hemodynamic profiles before and after immunosuppressive therapy until withdrawal of the intra-aortic balloon pump. CI: cardiac index, TnT: troponin T, SvO2: mixed venous oxygen saturation, DOB: dobutamine, IABP: intra-aortic balloon pump, IVIG: intravenous immunoglobulin, PSL: prednisolone, TAC: tacrolimus

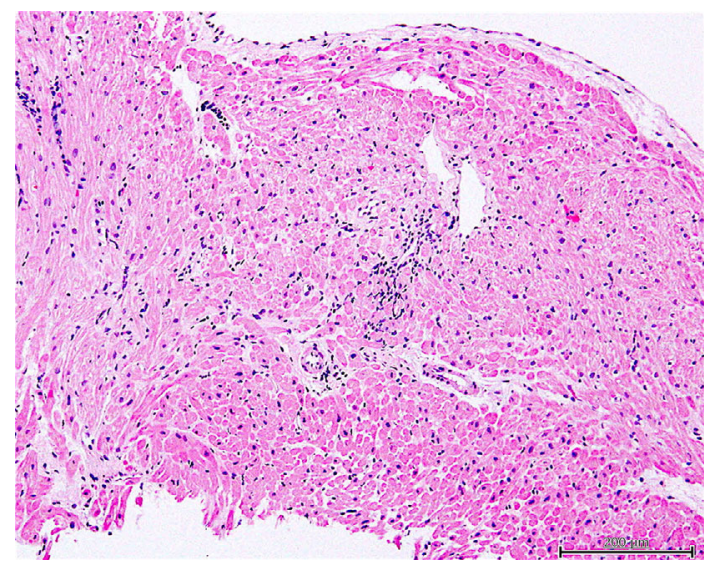

Figure 3. Histological findings from the right ventricular apex after treatment, demonstrating the absence of giant cell or eosinophil infiltration and only mild residual lymphocyte infiltration. Hematoxylin and Eosin staining.

Recently, a study related to solid-organ transplantation demonstrated a decrease in adverse effects and an increase in efficacy with tacrolimus/mycophenolate-based regimens compared with cyclosporine/azathioprine-based regimens (3). Furthermore, other studies have described several patients who were treated with antithymocyte globulin in addition to steroids, tacrolimus, and mycophenolate mofetil. In these patients, the dose of tacrolimus was adjusted so that the blood concentration was 8 to $12 \mathrm{ng} / \mathrm{mL}$ in the initial stage of administration and 6 to $8 \mathrm{ng} / \mathrm{mL}$ during the maintenance stage $(6,7)$. One of the merits of tacrolimus was that the drug efficacy could be monitored by measuring the trough value. Although the utility of IVIG therapy for acute myocarditis is controversial, a recent study showed that this therapy can significantly reduce in-hospital mortality and improve the LVEF of these patients (4).

To our knowledge, there have been no previous reports of patients treated with this regimen, and this report is the first to describe a patient with giant cell myocarditis who was discharged home without symptoms after receiving combination immunosuppressive therapy including tacrolimus. The dramatic clinical improvement was also confirmed by an increase in the $\mathrm{CI}$ and a decrease in the myocardial troponin $\mathrm{T}$ level. In addition, approximately 2 months after the administration of the combination therapy, the LVEF improved to $42 \%$, with a notable disappearance of the inflammatory cell infiltration in the myocardium, which was histologically confirmed by a repeated endomyocardial biopsy.

However, in biopsy-proven cases, the most frequent outcome is death or transplantation within the first year after the diagnosis. An elevated troponin T level $(>0.085 \mathrm{ng} / \mathrm{mL})$ and moderate to severe myocardial necrosis or fibrosis predicted a particularly high rate of early death or transplantation (8). Even with transplantation, Giant cell myocarditis recurrence in the donor heart has been reported to be as 
high as $20 \%$ to $25 \%$ (9). Combination therapy with steroid, tacrolimus, and IVIG may be an effective treatment option for patients with giant cell myocarditis, but careful followup of our patient will certainly be needed to truly assess the effectiveness of this treatment regimen. Finally, our local heart team decided to perform this treatment using the described regimen, and informed consent was obtained from the patient.

The authors state that they have no Conflict of Interest (COI).

\section{Acknowledgement}

We are deeply indebted to all of the members of the Sakakibara Intensive Heart Care Team for their sincere contributions to caring for this patient.

\section{References}

1. Cooper LT Jr, Berry GJ, Shabetai R. Idiopathic giant-cell myocarditis--natural history and treatment. Multicenter Giant Cell Myocarditis Study Group Investigators. N Engl J Med 336: 18601866, 1997.

2. Bang V, Ganatra S, Shah SP, et al. Management of patients with giant cell myocarditis: JACC review topic of the week. J Am Coll Cardiol 77: 1122-1134, 2021.
3. Kociol RD, Cooper LT, Fang JC, et al. Recognition and initial management of fulminant myocarditis: a scientific statement from the American Heart Association. Circulation 141: e69-e92, 2020.

4. Huang X, Sun Y, Su G, Li Y, Shuai X. Intravenous immunoglobulin therapy for acute myocarditis in children and adults. Int Heart J 60: 359-365, 2019.

5. Kandolin R, Lehtonen J, Salmenkivi K, et al. Diagnosis, treatment, and outcome of giant-cell myocarditis in the era of combined immunosuppression. Circ Heart Fail 6: 15-22, 2013.

6. Chaudhry MA, Correa A, Lee C, Yoon A, et al. Modern day management of giant cell myocarditis. Int J Cardiol 15: 82-84, 2015.

7. Steinhaus D, Gelfand E, VanderLaan PA, Kociol RD. Recovery of giant-cell myocarditis using combined cytolytic immunosuppression and mechanical circulatory support. J Heart Lung Transplant 33: 769-771, 2014

8. Ekström K, Lehtonen J, Kandolin R, et al. Long-term outcome and its predictors in giant cell myocarditis. Eur J Heart Fail 18: 1452-1458, 2016.

9. Maleszewski JJ, Orellana VM, Hodge DO. Long-term risk of recurrence, morbidity and mortality in giant cell myocarditis. Am J Cardiol 115: 1733-1738, 2015.

The Internal Medicine is an Open Access journal distributed under the Creative Commons Attribution-NonCommercial-NoDerivatives 4.0 International License. To view the details of this license, please visit (https://creativecommons.org/licenses/ by-nc-nd/4.0/).

(C) The Japanese Society of Internal Medicine Intern Med Advance Publication 